\title{
Robot Swarm Communication Networks: Architectures, Protocols, and Applications
}

\author{
Ming Li \\ Department of Computer Science \\ California State University, Fresno \\ Fresno, CA 93740, USA \\ mingli@csufresno.edu \\ Min Chen \\ Department of ECE \\ University of British Columbia \\ V6T 1Z4, Canada \\ minchen@ece.ubc.ca
}

\author{
Kejie Lu \\ Department of ECE \\ University of Puerto Rico at Mayagüez, \\ Mayagüez, Puerto Rico 00681 \\ lukejie@uprm.edu \\ Shiwen Mao \\ Department of ECE \\ Auburn University \\ Auburn, AL 36849, USA \\ smao@ieee.org
}

\author{
Hua Zhu \\ San Diego Research Center \\ San Diego, CA 92121 USA \\ hua.zhu@argonst.com
}

\begin{abstract}
There have been increasing interests in deploying a team of robots, or robot swarms, to fulfill certain complicated tasks such as surveillance. Since robot swarms may move to areas of far distance, it is important to have a pervasive networking environment for communications among robots, administrators, and mobile users. For this purpose, we propose to build a wireless mesh network as the wireless backbone within the areas of special interest. One or more robots can get connected with a nearby mesh router and access the remote server. Within each swarm, a self-organizing mobile ad hoc network is formed. With this type of robot swarm communication network, there are many important open issues to be addressed.
\end{abstract}

Keywords-robot swarm, wireless mesh networks

\section{INTRODUCTION}

There has been much concern regarding effective and efficient surveillance recently. Although lots of human efforts are made to ensure the safety at the borders such as dispatching more security guard forces and building walls along the borders, these enforcements demonstrate several critical disadvantages. Especially, the extremely high cost and lack of sufficient flexibility may eventually fail such efforts. In contrast, emerging networking technologies such as wireless mesh networks (WMNs) can help create virtual walls where human and wireless devices share important information to make appropriate actions, thereby greatly reduce the cost and improve the operational efficiency.

On the other hand, robot swarms have gained lots of attention in the past a few years. Despite of many complicated artificial intelligent algorithms proposed to improve the correctness and efficiency of decision making of a single robot, using a team of robots has many advantages. First, single robot may still not be intelligent enough to fulfill some tasks, especially in complicated geographical environments where multiple obstacles exist on the path of the robots. Second, fault tolerance is critical in some applications such as surveillance and military battlefield where a single robot may be damaged or die due to battery failure. Third, a swarm of robots is desired for accomplishing surveillance tasks difficult for human beings due to its low cost and flexibility. Finally, the idea of robot swarm is very natural since most complicated tasks such as moving around a big obstacle and cargo transportation require collaboration among different robots. Furthermore, with the cost of robots being significantly reduced to several hundred dollars, dispatching multiple robots is becoming a feasible scenario for a broader set of potential users. Therefore, we believe that integrating robot swarms with wireless mesh networks provides a new solution of effective and interactive border surveillance.

In this paper, we propose a new architecture called robot swarm communication networks. In this architecture, robots are clustered to one or multiple teams or swarms and each swarm can be monitored and controlled by some central servers through a wireless mesh backbone as well as Internet. Within each swarm, a self-organizing mobile ad hoc network is formed such that all robots are connected to all other robots despite of the movements. Meanwhile, mobile users can also monitor swarms through mobile devices such as laptops and PDAs and can take action immediately based on certain information collected. The monitoring module may include location, topology, and related information. Pictures and videos are also proposed to be streamed from robots to servers or mobile users for appropriate decisions.

The proposed architecture poses new challenges from both protocol design and software system development aspects. On the one hand, how to coordinate robots that move randomly and continuously is a critical issue. Related issues include collaborative object recognition, deployment, etc. On the other hand, how to develop a software system to efficiently monitor, coordinate, and control robot swarms is important for the system to be put in practice. In addition, streaming video from robots requires quality of service (QoS) support from the wireless mesh network backbone. 
This paper is organized as follows. Section II discusses related works in robot swarm algorithms, softwares, and QoS protocols. Section III proposes the robot swarm communication network architecture. Section IV discusses the challenges from network protocol aspect. Section V discusses the challenges from software development aspect and Section VI concludes this paper.

\section{RELATED WORK}

\section{A. Robot swarm coordination:}

Mondada and Pettinaro [2] proposed the concept of Swarm-Bot, a group of autonomous mobile robots called SBOTs. S-BOTs have a particular assembling capability that enables them to connect physically to each other. With around 10 to 30 S-BOTs being interconnected, SWARM-BOT becomes much more robust than single bots and can handle difficult tasks even in hard environment conditions. Poduri and Sukhatme [4] investigated the self-deployment of a mobile sensor network and proposed to maximize the area coverage of the network with a $\mathrm{K}$ neighbor constraint. The core idea of the scheme is to control the location of mobile sensors through two forces: $F_{\text {cover }}$ that causes nodes to repel each other to increase their coverage and $F_{\text {degree }}$ that causes nodes to attract each other to have more number of neighbors. A node stabilizes its position when the two forces are equivalent. Sheng and Yang [3] proposed a multi-robot exploration model and studied the coordination among the robots in a group. A distance based bidding algorithm was proposed to enforce the coordination. Also, a map synchronization algorithm was proposed to minimize the amount of information exchange when two sub-networks merge.

\section{B. Robot monitoring/control softwares:}

Correll and Sempo [1] developed SwisTrack, a new software for tracking multi-unit robots and biological behaviors. SwisTrack is a framework that enables users to remotely monitoring the movement of robots/insects. First, the video images of the swarm is captured with a camera and then segmented to identify the targets in the background. Then, these data are transmitted over TCP/IP to the user side for post-processing. SwisTrack significantly ease the task of small robot movements. However, it is only used for monitoring purpose and the use of camera limits its application to small areas. It is more desirable to have a framework that can be used in general scenarios such as outdoor surveillance. McLurkin and Smith [6] proposed a human-robot interface for control iRobot Swarm that includes a team of 112 individual robots. But the emphasis of the research is to interact with a group of robots through commands or input from game controllers and does not take care of the independent behavior of robots. MobileEyes [5] is a commercial GUI software for driving or controlling individual robot by issuing commands and displaying the video captured by the moving robot. However, MobileEyes does not handle the coordination and control of a robot swarm.

\section{QoS support in wireless ad hoc networks:}

Xue and Ganz [15] proposed Ad hoc QoS on-demand routing (AQOR), a QoS routing protocol with admission control enforced to support quality of service in multi-hop ad hoc networks. Yang and Kravets [14] proposed to enforce admission control by considering the contention of all nodes within the carrier sensing area. However, expensive power consumption is required to obtain neighboring information. Also, they did not consider the link rate and traffic for bandwidth estimation. Chen and Heinzelman [8] proposed to calculate available bandwidth by local estimation and message exchange among neighboring nodes. Then, they adopted the result proposed by $\mathrm{Li}$ and Blake [10] to account for intra-flow interference. However, this result is not accurate enough, especially when multi-rate is enabled. With multi-rate MAC, the distance of a link may be much smaller due to transmission with high data rate, resulting in more intra-flow interference. To consider both the mobility, multi-rate feature, and the multilink interference, Li and Prabhakaran [11] proposed route available bandwidth (RAB) and route reliability (RR) metrics when admission control decision is made.

Awerbuch and Holmer [7] proposed medium time metric (MTM) for better route selection in ad hoc networks. Based on the assumption of complete interference, MTM chooses the routes with minimum accumulate medium time consumption. To achieve this, each link is assigned a link weight, which is determined by the link rate and data packet. However, the assumption of complete interference makes it only appropriate for small networks. Also, link loss ratio is not considered. Draves and Padhye [9] further considered multi-rate capability and combine link capacity and ETX for better performance in multi-radio, multi-hop wireless mesh networks. They propose Weighted Cumulative Expected Transmission Time (WCETT) to account for the interference among links that use the same channel. Also, WCETT calculation can be tuned for various different QoS objectives.

\section{NETWORK ARCHITECTURE}

The architecture of the proposed robot swarm communication network is illustrated in Figure 1. Wireless mesh routers are deployed on the top of buildings, walls, or towers. Each mesh router consists of multiple antennas and can operate over multiple channels to improve the network capacity and coverage. Mesh routers form a wireless backbone, which is further connected to wired Internet through gateways and IP routers. One or more team of robots are equipped with one or more wireless adaptors which can communicate with mesh routers or other robots, digital video camera, and GPS. Each robot swarm maintains continuous connectivity within itself and periodically updates the collected information such as its location, picture, and video to some administrators that reside either locally or remotely. Meanwhile, users such as security guards drive around the area frequently, access wireless devices such as PDA or Palm PC, and monitor one or more robot swarms. If certain emergency is identified, a user can immediately take action. Due to the limited computational power of these devices on image/video analysis, a user may also get certain instructions from the administrators for appropriate actions. Altogether, the proposed architecture presents an interactive coordination framework for frequent monitoring, efficient collaboration, and fast reaction. Furthermore, since of mesh routers, servers, robots, and PDAs are all inexpensive and require minimum human labor, such architecture is feasible and very cost-effective. 


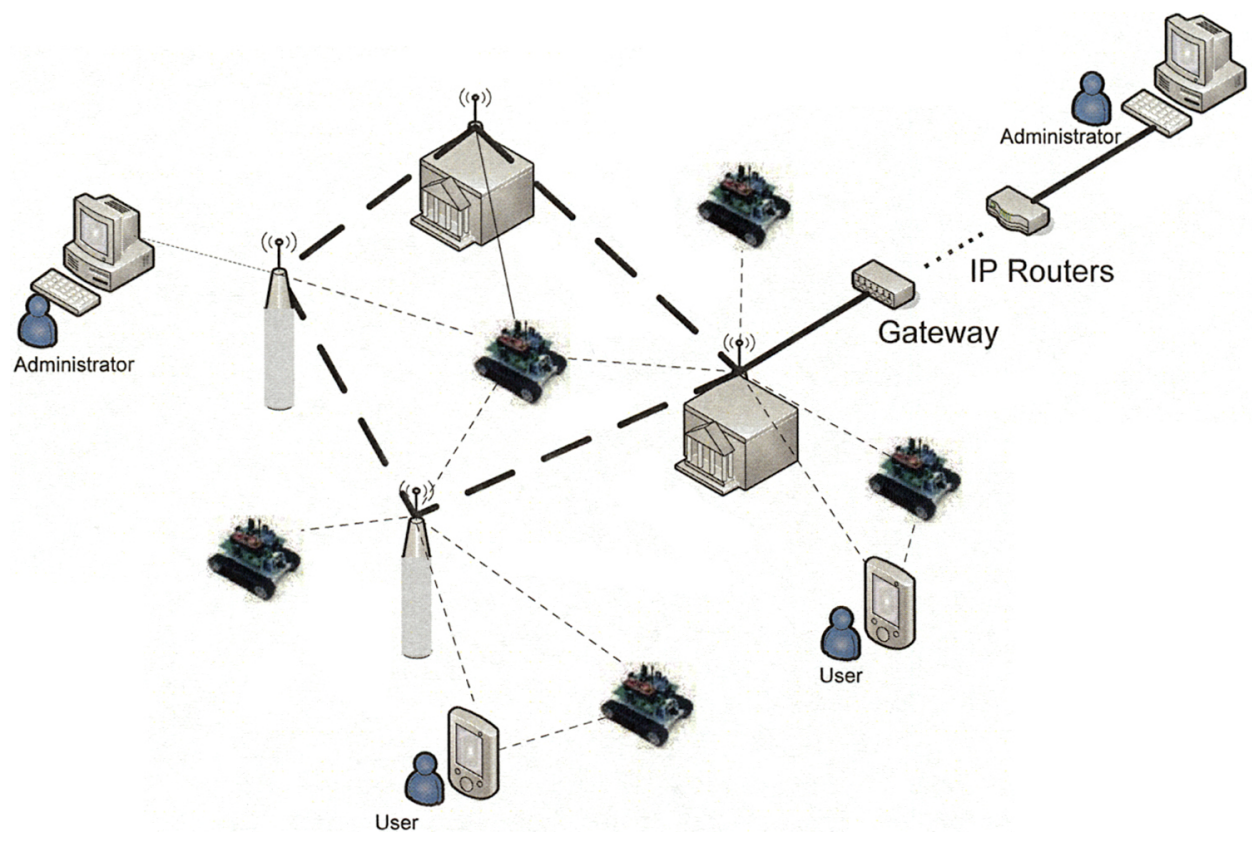

Figure 1. Architecture of robot swarm communication network

With the proposed architecture, the robot swarms help fulfill the following critical tasks difficult for humans:

- Continuous surveillance: a swarm of robots can move around various areas in a non-stopped pattern, which significantly improves the information accuracy and the timeliness of actions taken by security guards. Some robots can be made in very small size and cannot be easily detected to enhance the effectiveness of surveillance.

- Information collection: through effective coordination, a team of robots equipped with GPS, video camera, and sensors can capture image/video periodically, recognize sensitive objects such as enemy and chemical/biological stuff, and report to administrators or security guards instantly.

- Coverage inspection: a robot can report to the administrator immediately if it cannot receive wireless signal from the mesh routers, which helps identify certain areas subject to security problems.

Wireless mesh networks (WMNs) and mobile ad hoc networks (MANETs) have been investigated extensively. For WMNs, many schemes have been proposed to address issues such as wireless channel assignments, network capacity, and routing. For MANETs, many schemes have been proposed on mobility and quality of service (QoS) aware routing, energy efficient Medium Access (MAC) protocols, and topology control. Therefore, the emphasis of this research is not on WMNs and MANETs, but on the communications among administrators, users, and robot swarms. However, the mesh backbone provides an infrastructure to facilitate such communications with broadband capability.
With one or more teams of robots being dispatched, there are many interesting issues to be resolved. First, one important issue is how to monitor these swarms in a real-time manner. If robots can be effectively monitored, critical information such as the location of each robot, the connectivity, and other information can be collected at a central server. Based on the information from the swarm, the server may perform certain control and/or coordination to assist the robots if necessary. Second, how to design algorithms to allow intelligent robots to control and coordinate among themselves autonomously is a big challenge. The research on these topics, along with other related issues, is still in its infancy.

\section{PROTOCOL DESIGN CHALLENGES}

From the protocol design aspect, a set of protocols should be developed in following topics:

- Distributed coordination of robot swarms: where the problem of maintaining continuous connectivity is handled by incorporating network information such as topology, energy level, and link condition with robot status such as moving, working, or idle.

- QoS support in wireless mesh networks: where efficient protocols are designed to support voice/video streaming in multi-radio multi-channel multi-hop wireless mesh networks.

\section{A. Distributed coordination of robot swarms}

Usually robots need to exchange certain information such as location and status in order to make global decision. In this case, maintaining continuous connectivity of a swarm is critical for the successful collaboration among robots within a swarm. The key challenge of coordination is how to ensure swarm connectivity without the prior knowledge about robot mobility pattern, i.e., robots can move freely based on its own 
intelligence and tasks. Compared to centralized approaches where a central control robot coordinate the movement of other robots, distributed approaches exhibits several advantages such as better scalability, efficiency, and fault tolerance. Based on collected information from neighbors through periodical message exchange, a robot can determine how to guide its own movement to achieve certain goals while maintaining effective connectivity. In general, successful coordination protocols include, but not limited to the following:

- Movement based swarm coordination: It is desirable to have a distributed algorithm such that each node determines its moving speed and direction to avoid link breaking.

- Swarm partitioning recovery: Although coordination maximizes the connectivity, it is still possible that a swarm may get partitioned due to complicated geographical environments or one or more robots are attacked and damaged. A swarm partitioning recovery algorithm should be devised to first quickly detect the occurrence of the swarm partition. Then, based on the recorded historical connectivity knowledge, each robot decides its movement to recover the connectivity with previous neighbors.

- Energy efficient swarm coordination: Since each robot only has limited battery power, it is important to minimize energy consumption. While coordination algorithms try to minimize necessary movements, power adjustment technique can be used to minimize the required energy to maintain effective connectivity.

- Swarm deployment: robots may move from one location to another location to perform certain tasks. How to maximize an area with a given number of robots is an interesting issue. A successful deployment algorithm has to be able to adapt to various environmental constraints such as obstacles and walls.

- Data fusion and collaborative object recognition: Another important issue in robot swarm is how to make robots collaborate with each other to recognize certain targets. Due to the limited knowledge at each robot, it is critical for robots to share the information with each other and reach the same conclusion. Then, data are analyzed to decide their relative importance for sharing. Finally, a minimum data set selector is designed to choose to disseminate to neighboring robots.

\section{B. QoS support in robot swarm networks}

In wireless mesh networks (WMNs), multi-channel or multi-radio features are enabled at mesh routers. Similar to IEEE 802.11 WLAN, WMNs suffer from significant channel interference and thus not able to fully achieve its high capacity and support sufficient QoS for multimedia applications. The following topics should be investigated:

- Network reconfiguration: How to design an automatic network configuration protocol such that the IP address of a robot swarm is dynamically changed according to the associated mesh router.
- QoS support: How to design a wired/wireless integration scheme such as [12] to support end-to-end QoS support for users accessing Internet within a mesh network.

- Load balancing: The QoS experienced by wireless end users is constrained by the number of gateway routers and available channels. Therefore, it is desirable to balance the load of network traffics going through gateway routers. Furthermore, as users move, it might be more appropriate to route traffics to a closer gateway node or a gateway node with higher route available bandwidth.

\section{SOFTWARE SYSTEM DEVELOPMENT CHALLENGE}

Although several softwares [1][5][6] have been developed, they are either designed to monitor a single robot, or does not perform control and coordination, or are non-real time and used just for the purpose of simulation. To meet the software needs resulting from the increasing deployment of network enabled robot swarms, we have designed and developed ROBOTRAK [13], a secure software for monitoring, control, and coordination of intelligent robotic swarms. Using TCP connections through the wireless medium, the ROBOTRAK server can exchange information with the robotic swarm reliably and continuously. For monitoring purpose, all the bots collect and report wireless signal strength, interference, neighboring bots list, and location information. For control purpose, a new robot can be dispatched to join a swarm or an existing robot in a swarm can be guided to move to a specific destination. For coordination purpose, the server maintains the connectivity of the swarm under various situations. Furthermore, in order to maintain network privacy and avoid outsider intrusion, multi-security levels and a dynamic password technique were implemented.

The major advantages of ROBOTRAK are:

- Flexibility with comprehensive features on robot monitoring, control, and coordination.

- User friendliness with nice graphical user interface.

- Multi-level security at both application layer and transport layer.

- Robustness with the implementation of reliable socket communication.

ROBOTRAK can run on any server with Internet connection and requires the robots in a target swarm to be equipped with wireless adaptors (such as $\mathrm{WiFi}$ ) and global positioning system (GPS). All the messages can be transmitted/received through typical wired/wireless multi-hop network connections. The software was implemented with Microsoft Visual Basic.Net and can be easily modified to provide web-based versions. All the designed features and functionalities have been implemented with user friendly graphical user interfaces (GUI) and extensively tested in various scenarios such as multiple robots and multiple instances of running ROBOTRAK software. 
Right now, ROBOTRAK has provided basic functionalities for monitoring, coordination, and control of robot swarms. Many new features should be added to make it more practical. We intend to work on the following:

- Video streaming: We will implement the data communication and video rendering modules to enable video streaming from any robot to the server. To improve the quality, peer to peer techniques will be used.

- Robot movement guidance: Based on the location of the robots, the server can quickly look up the geographical information from the database and provide certain guidance on moving around the specific areas.

- Integration with coordination algorithms: We will implement various coordination algorithms in $\mathrm{T} 1$ to maintain effective connectivity within a swarm to build a heterogeneous system to take advantage of both central control from the server and the distributed coordination within the swarm.

\section{CONCLUSTION}

Robot swarm has emerged in recently years as a solution for surveillance in complicated geographical environments thanks to the significant cost reduction of individual robots and accessories such as wireless adaptors, GPS, and video cameras. With a team of robots being dispatched, several challenges arise. From the communications aspect, continuous network connectivity has to be maintained despite of random movement patterns and various status and roles of each robot. From the algorithms aspects, how to enable effective and efficient collaboration among robots for appropriate decision making is critical to the success of task fulfillment. On the other hand, it is imperative to design and develop a secure real-time software system such that administrators and mobile users can monitor, coordinate, and control robot swarms remotely.

\section{ACKNOWLEDGEMENT}

This work is supported by the Provost Activity Awards at California State University, Fresno. Shiwen Mao's research has been supported in part by the National Science Foundation under Grant ECCS-0802113, and through the Wireless Internet Center for Advanced Technology (WICAT) at Auburn University.

\section{REFERENCES}

[1] N. Correll, G. Sempo, Y. Lopez de Meneses, J. Halloy, J.-L. Deneubourg, and A. Martinoli, "SwisTrack: A Tracking Tool for MultiUnit Robotic and Biological Systems", in IEEE/RSJ International Conference on Intelligent Robots and Systems (IROS), pages 21852191, 2006.

[2] Mondada, F., Pettinaro, G. C., Guignard, A., Kwee, I., Floreano, D., Deneubourg, J.-L., Nolfi, S. and Gambardella, L.M., Dorigo, M. (2004) SWARM-BOT: a New Distributed Robotic Concept. Autonomous Robots, special Issue on Swarm Robotics, Volume 17, Issue 2-3, September - November 2004, Pages 193 - 221.
[3] W. Sheng, Q. Yang, J. Tan and N. Xi, Distributed Multi-robot Coordination in Area Exploration, Robotics and Autonomous Systems (Journal). Page: 945-955, Issue 54. 2006.

[4] Poduri, S. and Sukhatme, G. S. (2004). Constrained Coverage for Mobile Sensor Networks. IEEE International Conference on Robotics and Automation, New Orleans, LA, USA.

[5] MobileEyes: http://www.mobilerobots.com/MobileEyes.html

[6] James McLurkin, Jennifer Smith, James Frankel, David Sotkowitz, David Blau, Brian Schmidt. "Speaking Swarmish: Human-Robot Interface Design for Large Swarms of Autonomous Mobile Robots", AAAI Spring Symposium, March 28, 2006.

[7] B. Awerbuch, D. Holmer, and H. Rubens, "High Throughput Route Selection in Multi-Rate Ad Hoc Wireless Networks", in Proccedings of WONS’04, Madonna di Campiglio, Italy, January 2004.

[8] L. Chen, W. Heinzelman, "QoS-aware routing based on bandwidth estimation for mobile ad hoc networks", IEEE Journal on Selected Areas of Communication, Vol. 23, No. 3, March 2005.

[9] R. Draves, J. Padhye, and B. Zill, "Routing in multi-radio, multi-hop wireless mesh networks". In Proceedings of ACM MobiCom 2004, Philadelphia, PA.

[10] J. Li, C. Blake, D.S.J. De Couto, H.I. Lee and R. Morris, "Capacity of ad hoc wireless networks," ACM MobiCom'01, pp. 61-69, 2001.

[11] Ming Li, B. Prabhakaran, "On Supporting Reliable QoS in Multi-hop Multi-rate Mobile Ad Hoc Networks", Best Student Paper Award, in Proceedings of the First IEEE International Workshop on Next Generation Wireless Networks (WoNGeN'05), Goa, India, Dec. 18-21, 2005.

[12] Ming Li, Hua Zhu, Imrich Chlamtac, B. Prabhakaran, "End-to-end QoS Framework for Heterogeneous Wired-cum-Wireless Networks", ACM/Springer/URSI Wireless Networks (WINET), pages 439 - 450, Vol. 12, Issue 4, July 2006.

[13] Ming Li, Anthony Alvarez, Francesco De Pellegrini, Imrich Chlamtac, B. Prabhakaran, "Design and Development of A Secure Real-time Monitoring, Control, and Coordination Systems for Intelligent Robot Swarms", poster paper, ROBOCOMM 2007, October 2007.

[14] Y. Yang and R. Kravets, "Contention-Aware Admission Control for Ad Hoc Networks", UIUCDCS-R-2003-2337, May 2004.

[15] Q. Xue, A. Ganz, "Ad hoc QoS on-demand routing (AQOR) in mobile ad hoc networks," Journal of Parallel Distributed Computing, Vol. 63 , pp. 154-165, 2003. 